
\title{
3 Research Square \\ The "diagonal" principle in the surgery of femoral intertrochanteric fractures
}

\section{Guanghui Li ( $\sim 26954988 @ q q . c o m$ )}

First Traditional Chinese Medicine Hospital of Changde City https://orcid.org/0000-0002-2844-6405

Tuoen Liu

West Virginia School of Osteopathic Medicine

Jianhui Duan

First Traditional Chinese Medicine Hospital of Changde City

\section{Research article}

Keywords: intertrochanteric fracture, intramedullary fixation, technique

Posted Date: October 1st, 2020

DOl: https://doi.org/10.21203/rs.3.rs-78853/v1

License: (1) This work is licensed under a Creative Commons Attribution 4.0 International License.

Read Full License

Version of Record: A version of this preprint was published at Asian Journal of Surgery on September 1st, 2021. See the published version at https://doi.org/10.1016/j.asjsur.2021.08.029. 


\section{Abstract}

Objective: To explore the role of "diagonal principle" surgical technique in the treatment of elderly femoral intertrochanteric fractures.

Methods: From October 2015 to February 2018, we treated 28 cases of femoral intertrochanteric fractures ( 8 cases of AO / OTA classification 31A1 type, 12 cases of 31A2 type, and 8 cases of 31A3 type), and they all received extended pith internal nail (length $\geq 340 \mathrm{~mm}$ ) internal fixation surgery.

Results: Hip function scores were excellent in 18 cases, good in 7 cases, poor in 1 case, and dead in 2 cases. The excellent and good rate was $89.3 \%$. No internal fixation fracture, failure, or re-fracture occurred.

Conclusion: The use of "diagonal principle" for the treatment of elderly femoral intertrochanteric fractures has promising results.

\section{Introduction}

Intramedullary nail fixation has become of the primary treatments for intertrochanteric fractures, which are more commonly seen in the elderly. ${ }^{[1]}$. Determining the appropriate surgical method of repair for intertrochanteric fractures has profound effects on patientquality of life and long-term survival rate, especially in geriatric populations. Although the application of short intramedullary nails is adequate in most cases; some A2 patients and A3 patients, identified through the AO/OTA fracture classification, requirelong intramedullary nails for proper fixation ${ }^{[2]}$. The short intramedullary nail tip is clinically valued because it is able toindert into theanterior cortex of the distal femur (Figure $1 \mathrm{a}$ and $1 \mathrm{~b}^{[3]}$. The long medullary nail is able to reach the anterior cortex of the distal femur, however, it can also inadvertently extend beyond this region ${ }^{[4]}$. The nail tip of the long intramedullary nail can extend too far anteriorly (Figure $2 \mathrm{a}$ and $2 \mathrm{~b}$ ), which can stimulate the anterior cortex during hip flexion, causing pain to the patient andincreasing the risk of fracture around the tip of the intramedullary nail. Many doctors attribute this occurrence to the curvature of the intramedullary nail and may even consider this to be an unavoidable event associated with the use of long intramedullary nails ${ }^{[5]}$. However, we believe that proper positioning of the needle point can help to avoid this situation. The needle insertion point affects the position of the nail tail. The forward needle insertion point will center the nail tail or position it slightly backward and the backward needle insertion point will cause the nail tail to be positioned toofar forward. This theory is similar to the "diagonal" principle of a rectangle, The diagonal of a rectangle is a diagonal line segment from point $A$ to point $B$ of the rectangle. Duringn the surgery, if we regard the sagittal plane of femoral shaft medullary cavity as a rectangle, so when the intramedullary nail is implanted from the backward entry point $(A)$, because the intramedullary nail cannot be bent, the tail of the intramedullary nail will enter point $B$ along the diagonal line, which will make the tail of the intramedullary nail move to anterior cortex of femur(point B). (Figure 3). 


\section{Clinical Data And Methods}

Clinical data: General Information:Data collection occuredfrom October 2015 to February 2018, and included data from patients with surgically corrected intertrochanteric fractures.. Participation in the study was determined based on the following inclusion criteria: (1) Clear history of trauma; (2) Age 62 to 95 years; (3) Imaging diagnosis of fresh intertrochanteric fractures (AO / OTA classification of 31-A1, 31A2, 31-A3) (4)Internal fixation surgery with long intramedullary nail .Exclusion criteria: (1) Pathological fracture or old fracture (injury time $\geq 3$ weeks); (2) Under 60 years old ( $<60$ years old); (3) Multiple fractures; (4) Additional surgery other than intertrochanteric internal fixation and subsequent hospitalization for surgery besides internal fixation surgery; (5)Internal fixation surgery with short intramedullary nail.Patient information:28 patients including 10 males and 18 females with age ranging from 62 to 95 years old, with an average of 78.4 years. There were 16 cases with left sided intertrochanteric fracture and 12 cases with right sided intertrochanteric fracture. The fractures were classified according to AO/OTA, including 8 cases of type $31 \mathrm{~A} 1,12$ cases of type $31 \mathrm{~A} 2$, and 8 cases of type $31 \mathrm{~A} 3$. The injury mechanism included 3 cases of traffic injuries, 23 cases of falls when walking, and 2 cases of falling from high altitude(Table 1 ).

Table 1

\begin{tabular}{|l|l|l|l|l|l|l|l|}
\hline male & femal & $60-70$ & $71-90$ & $\square 90$ & Traffic & Fall & $\begin{array}{l}\text { High } \\
\text { falling }\end{array}$ \\
\hline $10(35.7 \%)$ & $18(64.3 \%)$ & $9(32.1 \%)$ & $15(53.6 \%)$ & $4(14.3 \%)$ & $5(17.9 \%)$ & $17(60.7 \%)$ & $6(21.4 \%)$ \\
\hline
\end{tabular}

Table 2

\begin{tabular}{|l|l|l|l|l|l|}
\hline Harris score & $\begin{array}{l}\text { excellent } \\
(90-100)\end{array}$ & $\begin{array}{l}\text { good } \\
(80-89)\end{array}$ & $\begin{array}{l}\text { fair } \\
(70-79)\end{array}$ & $\begin{array}{l}\text { poor } \\
(\leq 69)\end{array}$ & dead \\
\hline result & $12(42.9 \%)$ & $6(21.4 \%)$ & $7(27.0 \%)$ & $1(3.8 \%)$ & $2(7.7 \%)$ \\
\hline
\end{tabular}

Treatment: Preoperative preparation: Before hospital admission, all patients were examined in the emergency department with pelvic orthotopic lateral X-ray (Figure $4 \mathrm{a}$ and $4 \mathrm{~b}$ ) and the intertrochanteric fracture classification was determined. Routine blood work, , liver and kidney function, blood coagulation testing, and blood typing were also performed in the emergency room20 patients underwent emergency surgery within 24 hours after their injury, 6 patients underwent surgery within 36 hours after their injury, 
and 2 patients underwent surgery within 72 hours after their injury ( 2 patients were complicated with diabetes, and it was necessary to control their blood sugar level prior to surgery). operation method:All patients underwent surgery on the orthopedic traction bed. The affected limbs were simultaneously adducted, the lower leg was internally rotated, and the fracture end was restored under C-arm fluoroscopy. When we confirmed the lateral position, we used a medical tape to attach a 2.0 Kirschner wirethat helped to mark the position of the needle on the skin (Figure5).After the surgical field was disinfected, a sterile towel sheet was placed. The proximal tip of the femoral trochanter is about $5.0 \mathrm{~cm}$ along the axis of the mark, and an approximately $3.0 \mathrm{~cm}$ surgical incision was made in this location. After exposing the fascia, the incision was sharply cut and a vascular clamp was applied.Fingertip palpation was utilized to locate the frontal area of the apex of the large trochanter, and the guide pin was drilled along the direction of the body surface marker. After drilling the guide pin, confirmation of itsposition at the large trochanter from the positive side was determined under fluoroscopy. If only minor adjustment was required, the openerwas used (Figure6) to adjust the guide pin insertion. If fluoroscopy revealed that the guide needle needed to be adjusted inward, the operator slowly rotatedthe tip of the opener inward, which can enlarge the inner bone tunnel. If lateral fluoroscopic imaging revealed that the guide needle needed to be adjusted to the front, the operator slowly rotated the tip of the opener forward, which can enlarge the bone tunnel in front.According to the standard surgical procedure ${ }^{[6]}$, when guiding the lateral position, the guide needle needs to be located at the front $1 / 3$ of the proximal tip of the femur trochanter. However, we moved this point forward by $1-2 \mathrm{~mm}$, because according to the diagonal principle (Figure7), this method can avoid the compression of the anterior cortex at the distal end of the femur after the intramedullary nail is implanted. After the femoral trochanteric tunnelwasmade using an electric drill, the guide needle was removed and the guidewire was implanted along the bone tunnel, and the appropriate intramedullary nail was selected according to the length of the patient's femur. A guide needle was drilled in the long axis of the femoral neck, and then anteroposterior and lateral fluoroscopic imaging was used to confirm appropriate positioning of the guide needle. After confirming the $\operatorname{TAD}$ of the guide pin $<10$, the main nail and the lower lag screw were respectively implanted.When locking the distal end of the intramedullary nail, we followed the diagonal principle. After selecting the appropriate length of the intramedullary nail, we immediately used the positioner for general positioning in vitro (Figure 8). After the positioning was completed, the distal locking pin only had an error in the front-rear direction (Figure 9).The inner diameter of the distal sleeve was $4.5 \mathrm{~mm}$. We used a $2.5 \mathrm{~mm}$ Kirschner wire to drill through one side of the cortical bone from the center of the sleeve. The sleeve was then removed and the C-arm was placed in a lateral position so that the position of the Kirschner wire relative to the intramedullary nail could be confirmed.If the Kirschner wirewas found to be behindthe nail, we withdrew the K-wire. Then drilled this Kirschner pin under the sleeve in an obliquely upward direction (Figure 10 and 10b). Using this method, (the diagonal principle), the needle insertion point will be higher, so the 2.5 gram needle will generally enter the lock hole (Figure 11).Once again, it was confirmed that the Kirschner wire was placed in the intramedullary nail hole and then we drilled through the contralateral cortex. While maintaining the position of the Kirschner wire, we insertedanother lock nail after drilling. After removing the $2.5 \mathrm{~mm} \mathrm{~K}$ wire, the locking operation wasperformed in sequence. This approach limited the time it took to obtain fluoroscopy whilethe oblique perspective of the C-arm ensured a successful operation(Figure 12 and 
12b). Rehabilitation after surgery: After waking from anesthesia, the patient began passive and active activities on the affected limb. On the first day after surgery, the patient was assisted in partially weight bearing on the affected limb while walking. Total weight bearing on the affected limb was attempted two weeks post-operation.

\section{Results}

26 patients were followed up at least 1 year post operation, (follow-up period between 12 to 15 months) with an average follow-up time of 13.8 months. Two patients died during the follow-up period. At the time of follow-up, hip function was scored using the Harris hip function score, w which is out of a score of 100 possible points ${ }^{[7]}$. Scores indicated hip function through the following scale: Excellent $\geq 90$ points; good: $\geq 80$ points; fair: $\geq 70$ points, the poor $\leq 69$ points. At the last follow-up, patients were excellent in 18 cases, good in 7 cases, poor in 1 case, and died in 2 cases. The excellent and good rate reached $89.3 \%$. There were no reports of incision skin necrosis, infection, loosening of the internal fixation or refractures. One of the patients had fat liquefaction after the incision. The incision healed smoothly after dressing and delayed suture removal.

\section{Discussion}

Choosing between long or short nails during internal fixation for intertrochanteric fractures in the elderly is still controversial. In 2016, John et al ${ }^{[8]}$. reported that short nails had a lower reoperation rate than long nails, and that short nails were associated with a shorter operation time, , less intraoperative blood loss, and lower hospitalization costs. Bovbjerg et al. claim that individual studies or meta-analysis show thatthere is no difference in the rate ofpost-operative complication leading to reoperation associated with short versus long nails [OR $0.89(0.49 ; 1.16)$ ]. They reported that there is no difference in the length of hospital stay between the two nail cohorts but did find that inserting a short nail took less operating time than insertion of a long nail $(p<0.0001)$. In the meta-analysis, they found no difference in 1-year mortality [OR $1.20(0.80 ; 1.79)]^{[9]}$. Hulet DA et al. also believe that patients undergoing long and short intramedullary nails have no significant difference in their complications and failure rates during followup $^{[10]}$. Although Hulet DA et al. report that the use of short and long nails for patients with31-A1 and 31A2 fractures is interchangeable ${ }^{[11]}$, we have found that long nails are recommended in patients with a large medullary cavity (diameter $>12 \mathrm{mmBecause}$ these patients belong to type 31-A2 fractures, short nail fixation may heal a fracture 3 months after post operation, but they pose a higher risk of being misplaced outward, subsequently stimulating the lateral femoral cortex (Figure 13). When we started using long intramedullary nails, we found that the position of the tail of the intramedullary nail at the distal end of the femur was often forward, and sometimes even stimulated the anterior cortex of the femur, causing postoperative pain and even local refracture. This problem has also been reported before, but was attributed to the design of the intramedullary nail and the curvature of the femur ${ }^{[12][13][14]}$. We postulate that this occurrence is mainly caused by the backward position of the needle insertion point, and as such, this problem can be solved by changing the insertion point of the intramedullary nail. Because the long 
intramedullary nail has a forward arc, we can imagine the lateral X-ray image of the femur as a rectangle. When implanting the longintramedullary nail, if the point of the needle is behind, the nail tail can easily impact the cortex in front of the femur (Figure 2a and2b). And if you advance the needle point as far as possible, the nail tail will be centered, or a little backward. This is similar to the "diagonal" principle of a rectangle.

The distal locking operation of long intramedullary nails often fails, which is one of the conditions that often troubles the surgeon during the operation. This also limits the application of long intramedullary nails ${ }^{[15]}$. In our surgeries, we found that the "diagonal" principle can be used when locking the distal nail, which expands the utility of long intramedullary nails. For our geriatric patient group, we chose long intramedullary nails for fixation and utilized our operative method discussed in the "operation method" section of this paper. After at least 1 year of follow-up, we found no fractures of the distal end of the intramedullary nail. Moreover, in all patients' follow-up X-ray films, the intramedullary nailing tail was located at the center of the medullary cavity or slightly behind (Figure 15), and no tailing was found.

It is worth mentioning that we used $y-\rrbracket$ intramedullary nails (AOSIMAI China). The operation of the proximal femoral head and neck screws is similar to that of InterTAN. After driving the guide needle, if you find that the TAD is not perfect, it is very difficult to adjust it slightly. When drilling the lower nail, you can adjust it accordingly to the front or the back of the guide needle. For example, if the guide pin is slightly forward, the outer bracket is moderately raised and then drilled, so that the lower screw is slightly backward(Figure16), and the TAD of the main screw can be slightly corrected.Before making a skin incision, we stick a Kirschner wire mark on the skin with an adhesive tape when seeing the lateral position(Figure 5). This can roughly mark the position of the incision in the anterior-posterior direction and the longitudinal axis of the femoral shaft, which is beneficial to control the direction of the guide needle and the forward and backward position of the insertion point. Although this is a small skill, in our experience, it can greatly reduce the operation time and reduce the radiation exposure time during the operation.

\section{Declarations}

\section{Acknowledgements}

Not applicable.

\section{Authors' contributions}

Designed research: Guanghui Li, Jianhui Duan

Performed research: Guanghui Li, Jianhui Duan

Collected data: Guanghui Li, Jianhui Duan

Analyzed and interpreted data: Guanghui Li, Jianhui Duan, Tuoen Liu 
Performed statistical analysis: Guanghui Li, Jianhui Duan

Wrote the manuscript: Guanghui Li, Jianhui Duan, Tuoen Liu

\section{Funding}

The clinical research projects were supported by Hunan Administration of traditional Chinese Medicine (NO: 201872)

\section{Availability of data and materials}

Not applicable.

\section{Ethics approval and consent to participate}

The study protocol (reference number: 2015,05,12) was reviewed and approved by the Medical Ethics of Committee of the First Traditional Chinese Medicine Hospital of Hunan Changde (Changde, Hunan, China). The study was carried out in accordance with the Declaration of Helsinki and amendments, and informed written consent was obtained from all subjects prior to enrollment.

\section{Consent for publication}

Consent for publication was obtained from every individual whose data are included in this manuscript.

\section{Competing interests}

The authors declare that they have no competing interests.

\section{References}

1. R. Socci Implant options for the treatment of intertrochanteric fracturesof the hip:rationaleevidenceand recommendations. Bone JointJ.2017Jan;99-B(1):128133.doi:10.1302/0301-620X.99B1.BJJ-2016-0134.R1.

2. Browner BD, Jupiter JB, Krettek C, Anderson PA. Intertrochanteric Hip Fractures. In: Skeletal Trauma: Basic Science, Management, and Reconstruction. Amsterdam: Elsevier, 2015:1683-1720.

3. Palm H, Jacobsen S, Sonne-Holm S, Gebuhr P, Hip Fracture Study Group.Integrity of the lateral femoral wall in intertrochanteric hip fractures: an important predictor of a reoperation. $J$ Bone Joint Surg [Am] 2007;89-A:470-475.

4. Chen F, Wang Z, Bhattacharyya T. Convergence of outcomes for hip fracture fixation by nails and plates. Clin OrthopRelat Res 2012;471:1349-1355.

5. Utrilla AL, Reig JS, Muñoz FM, Tufanisco CB. Trochanteric gamma nail and compression hip screw for trochanteric fractures: a randomized, prospective, comparative study in 210 elderly patients with a new design of the gamma nail. J Orthop Trauma 2005;19:229-233. 
6. Baumgaertner MR, Curtin SL, Lindskog DM, Keggi JM. The value of the tip-apex distance in predicting failure of fixation of peritrochanteric fractures of the hip. J Bone Joint Surg Am. 1995;77(7):1058-1064. doi:10.2106/00004623-199507000-00012

7. Harris, WH. Traumatic arthritis of the hip after dislocation and acetabular fractures: treatment by mold arthroplasty. An end-result study using a new method of result evaluation. J Bone Joint Surg Am 1969; 51: 737-755.

8. Dunn J, Kusnezov N, Bader J Long versus short cephalomedullary nail for trochanteric femur fractures (OTA 31-A1, A2 and A3): a systematic review. J OrthopTraumatol. 2016 Dec;17(4):361-367. Epub 2016 Apr 19. Review. PMID: 27093971

9. Bovbjerg $P$, Froberg $L$, Schmal $H$ Short versus long intramedullary nails for treatment of intertrochanteric femur fractures (AO 31-A1 and AO 31-A2): a systematic review. Eur J Orthop Surg Traumatol. 2019 Dec;29(8):1823-1831. doi: 10.1007/s00590-019-02495-3. Epub 2019 Jul 18.

10. Hulet DA, Whale CS, Beebe MJ 2019 Mar 1;42(2):e202-e209. doi: 10.3928/01477447-20190118-03. Epub 2019 Jan 31.

11. Kleweno C, Morgan J, Redshaw J, et al. Short versus long cephalomedullary nails for the treatment of intertrochanteric hip fractures in patients older than 65 years. J Orthop Trauma 2014;28:391-397.

12. R Luque, B Rizo, C Arvinius, AO/OTA 31-A2 Fractures: Long or Short Gamma Nail? Biomed J Sci \& Tech Res DOI: 10.26717/BJSTR.2017.01.000539 Volume 1- Issue 6 : 2017

13. Boone $C$, Carlberg KN, Koueiter DM, et al. Short versus long intramedullary nails for treatment of intertrochanteric femur fractures (OTA 31-A1 and A2). J Orthop Trauma 2014;28:96-100.

14. Kanakaris NK, Tosounidis TH, Giannoudis PV. Nailing intertrochanteric hip fractures: short versus long; locked versus nonlocked. J Orthop Trauma 2015;29:S10-S16.

15. Kleweno C, Morgan J, Redshaw J, et al. Short versus long cephalomedullary nails for the treatment of intertrochanteric hip fractures in patients older than 65 years. J Orthop Trauma 2014;28:391-397.

\section{Figures}



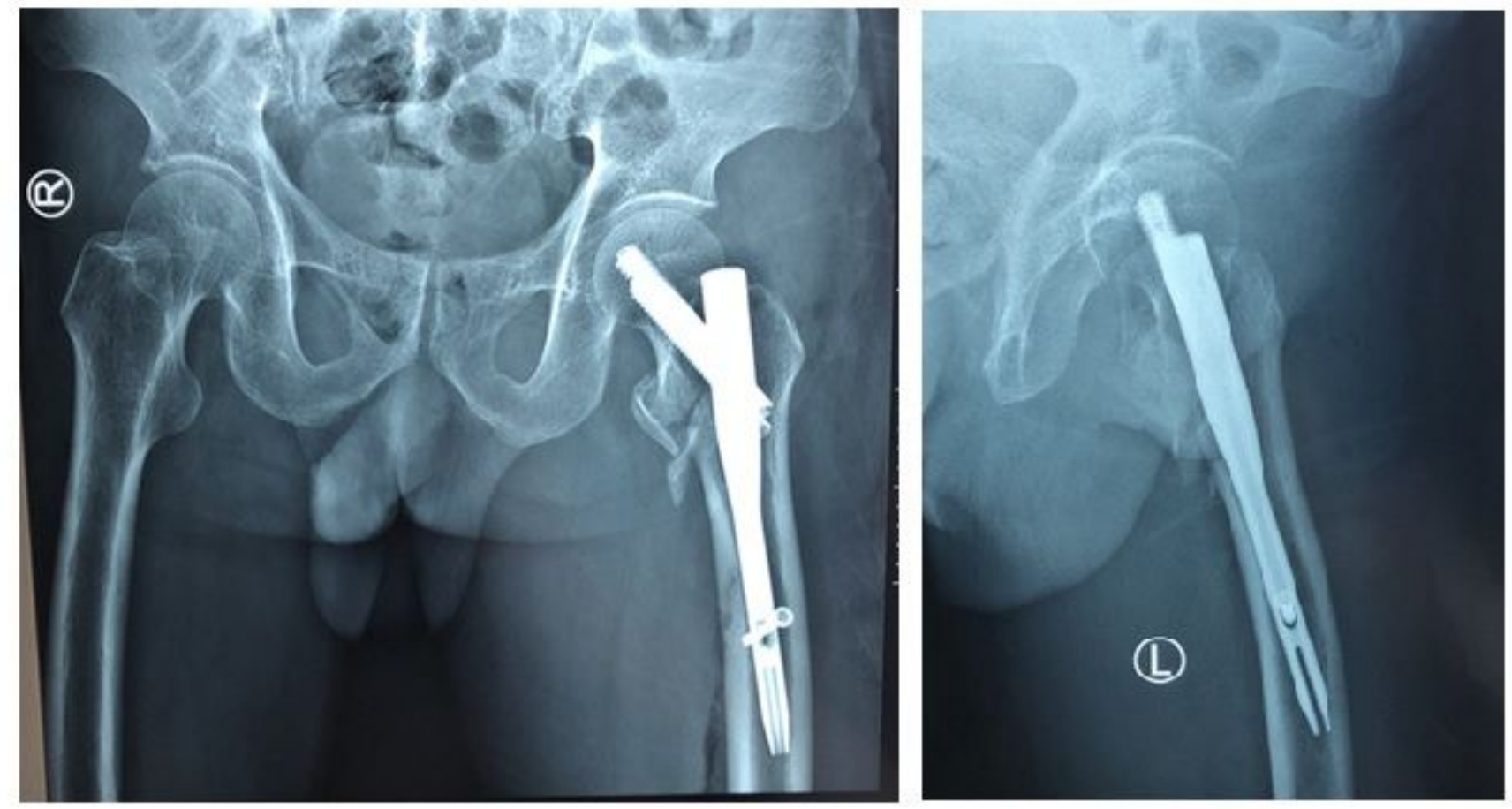

Figure 1

(captions not provided) 

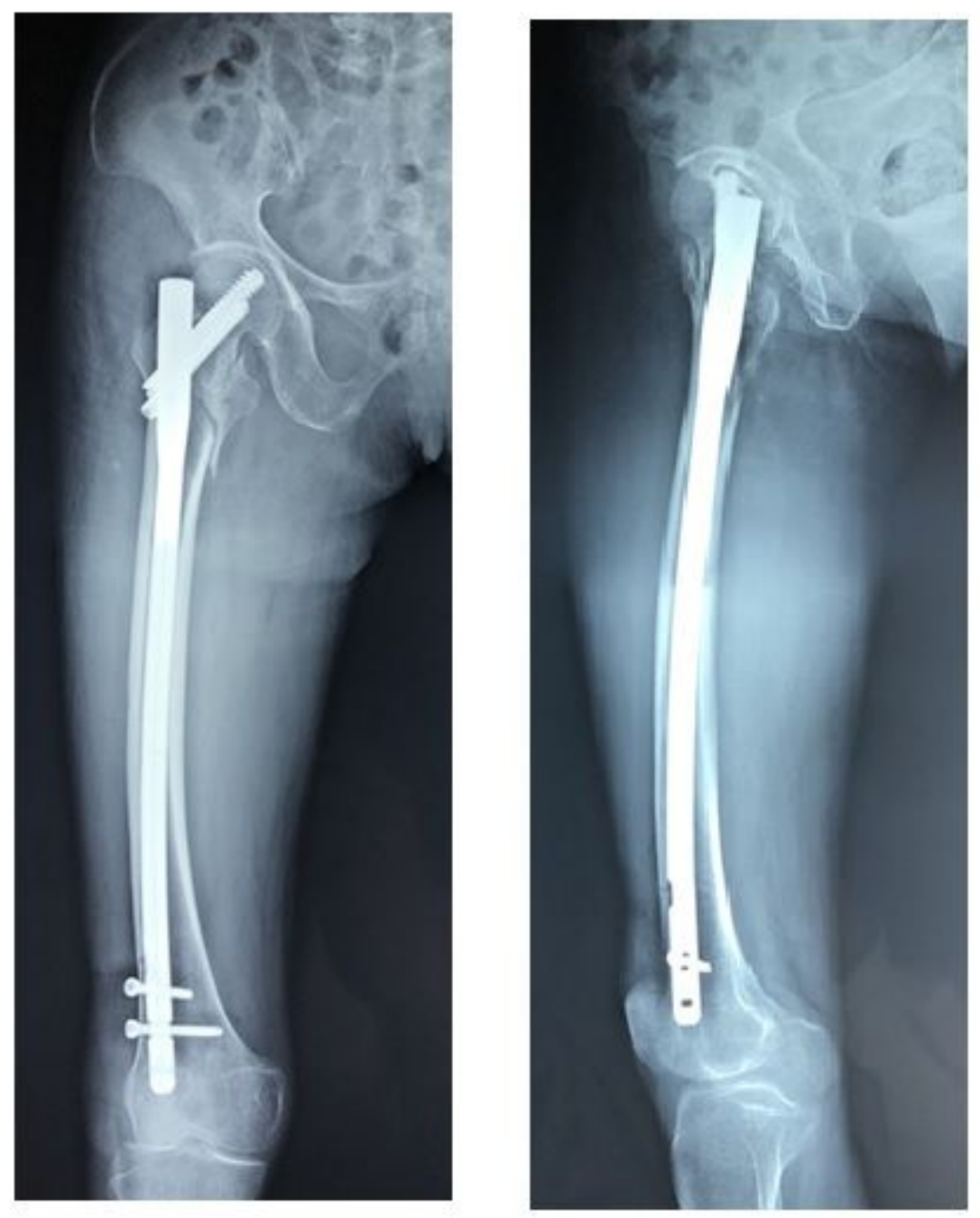

Figure 2

(captions not provided) 

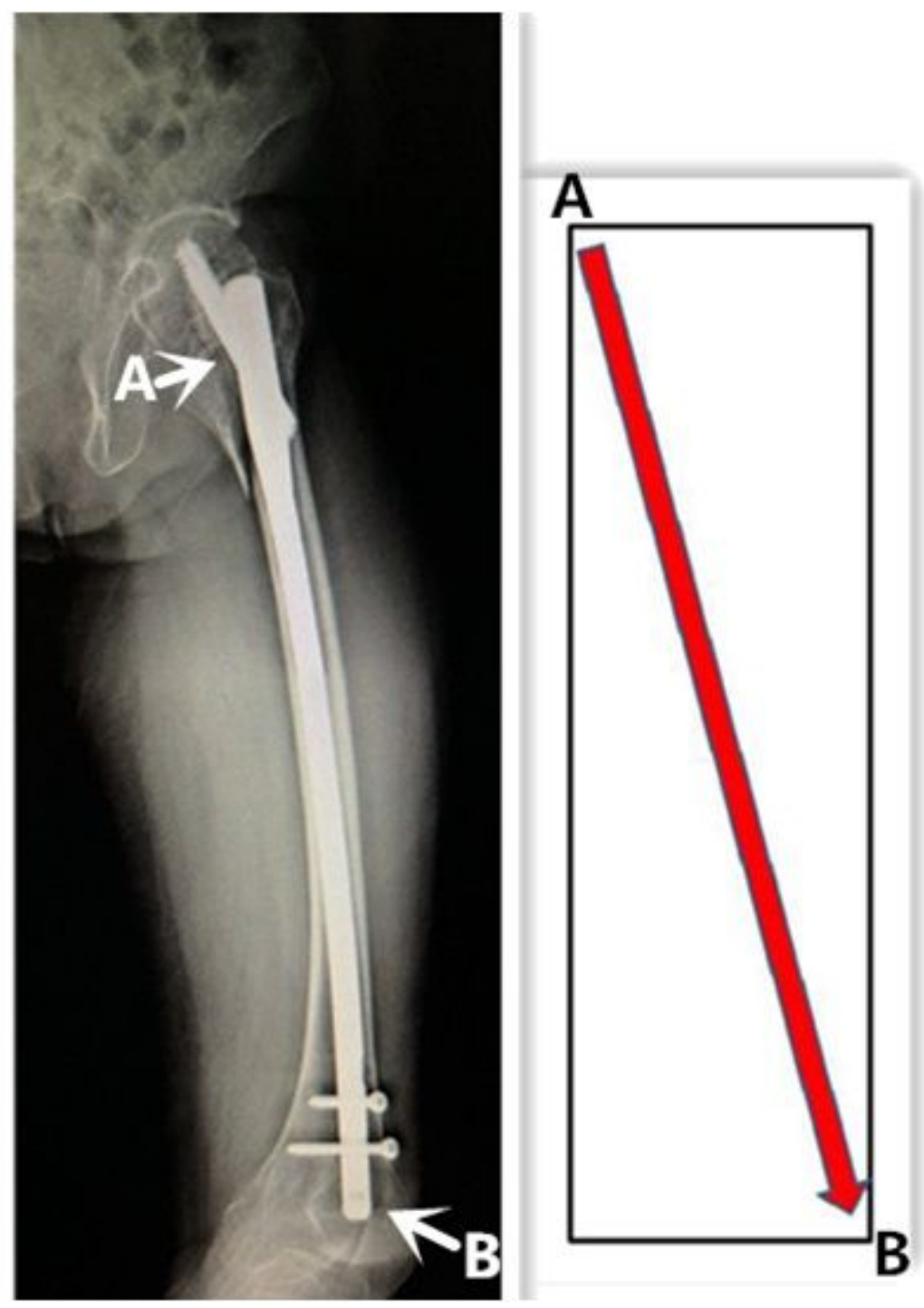

Figure 3

(captions not provided) 

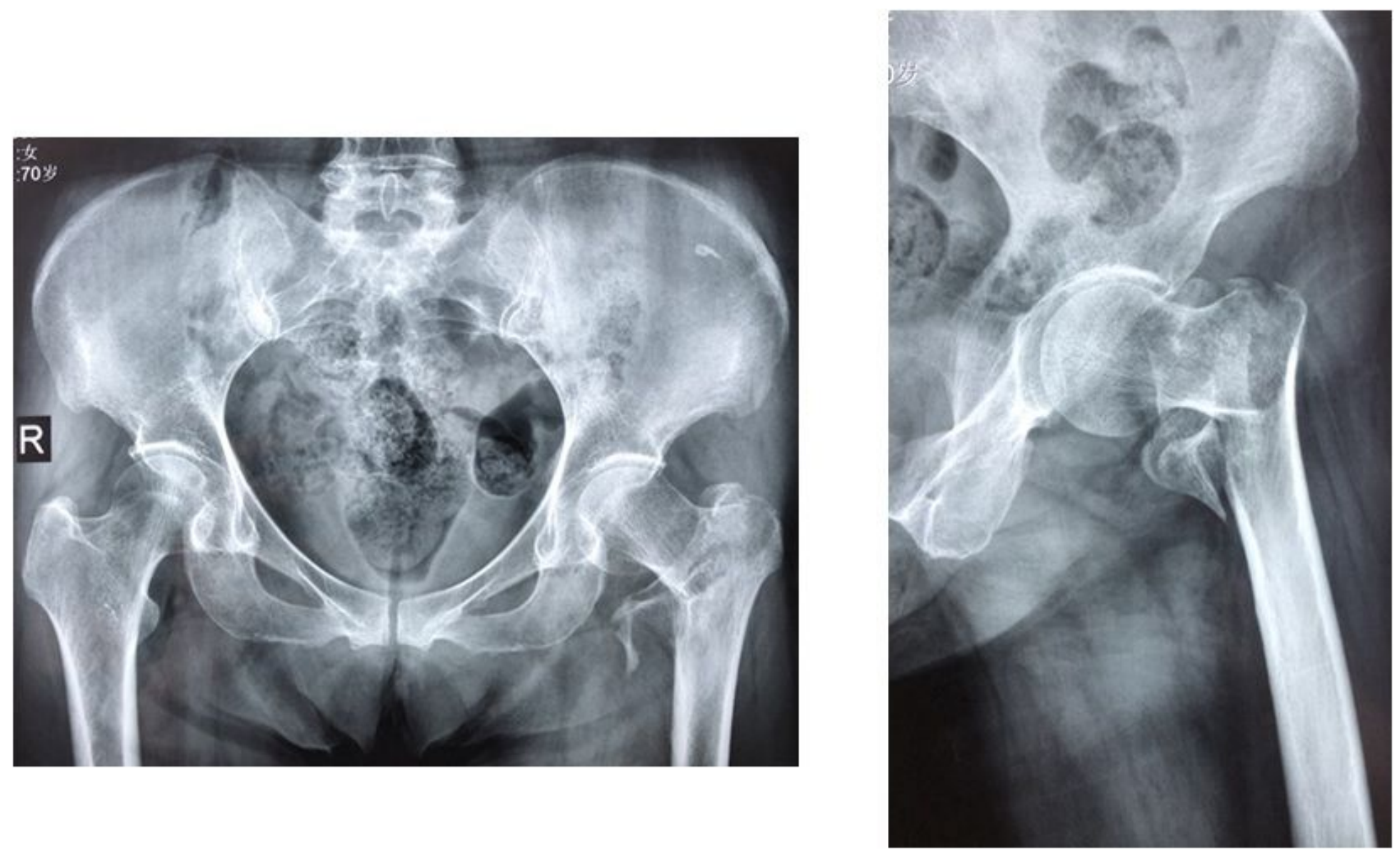

Figure 4

(captions not provided)

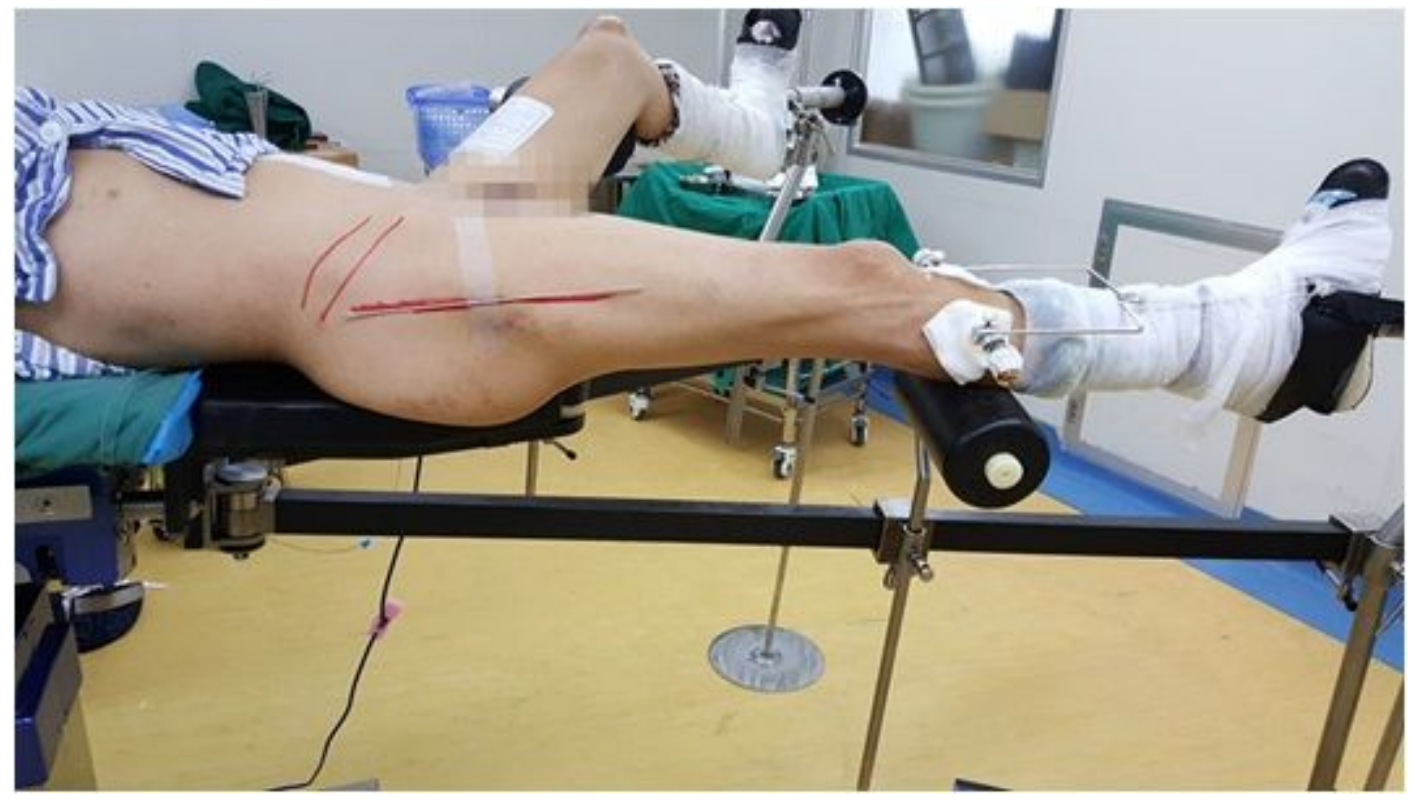

Figure 5

(captions not provided) 


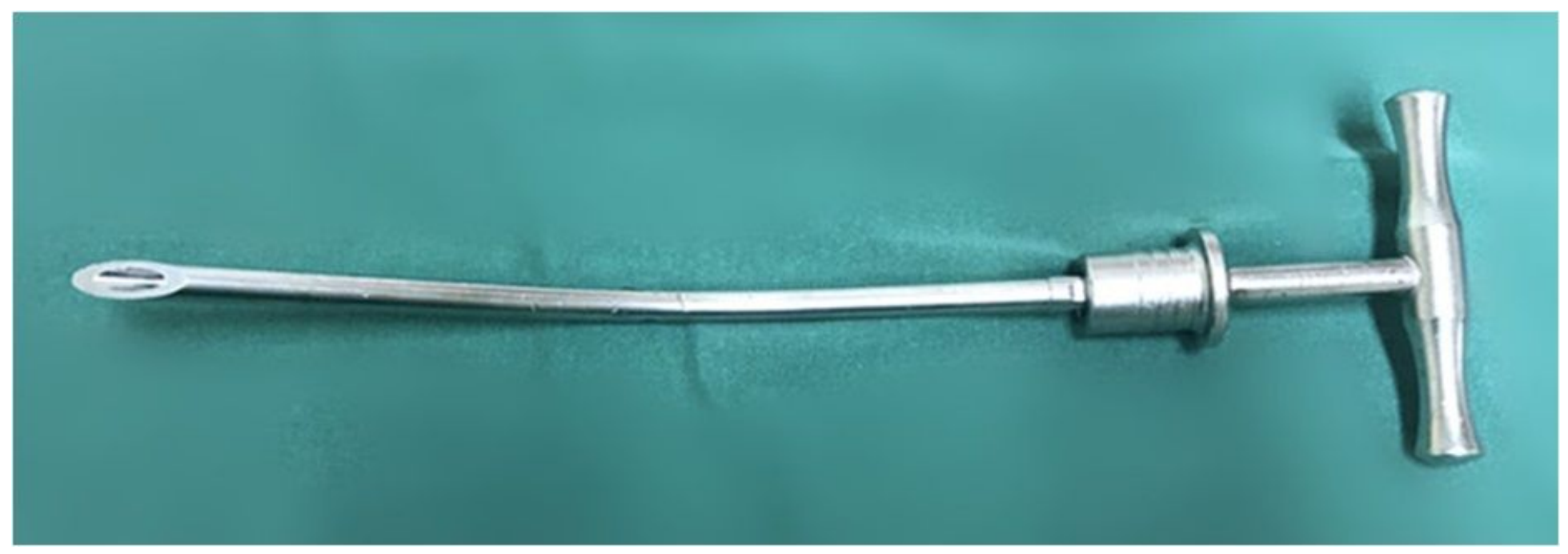

Figure 6

(captions not provided)
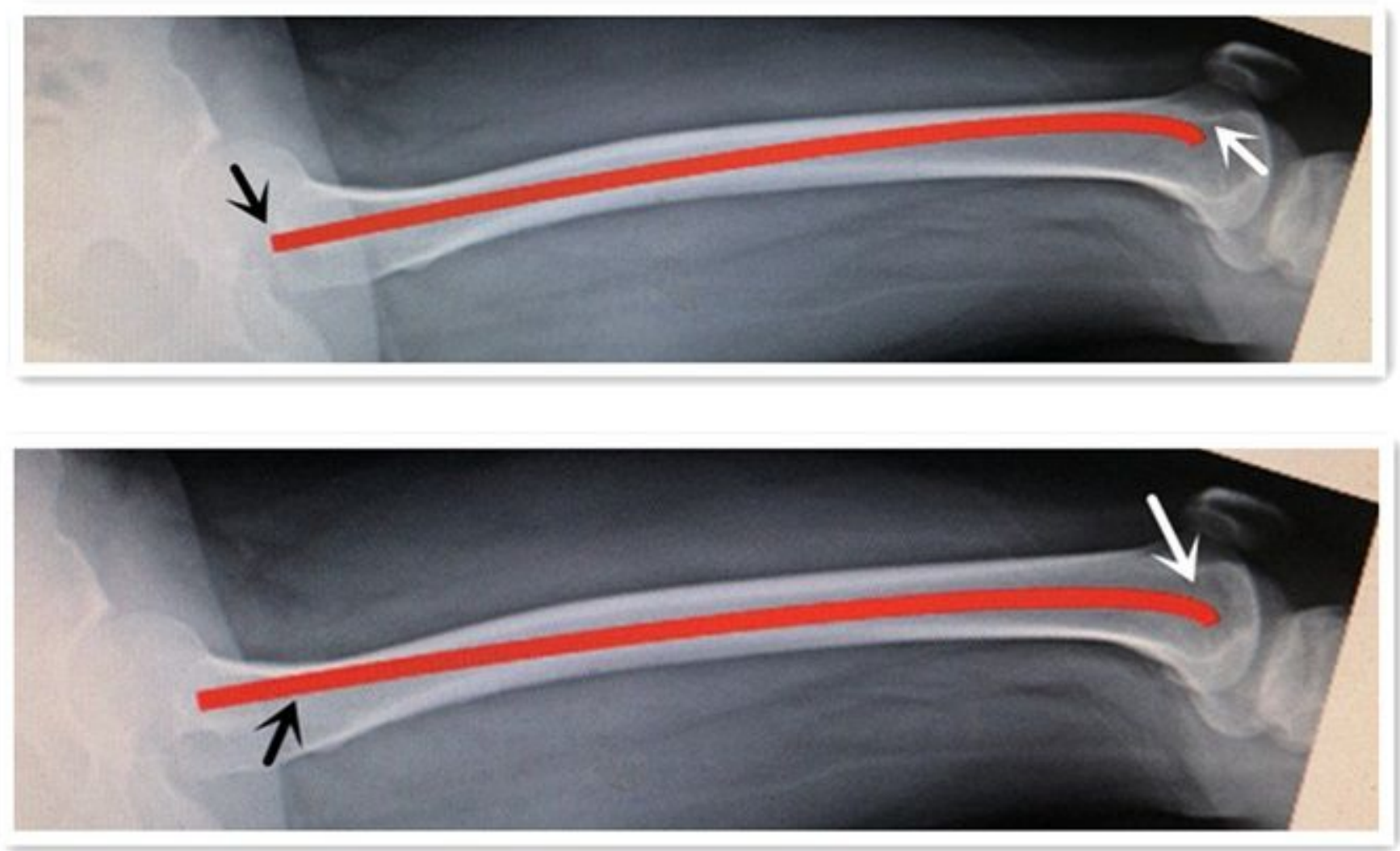

Figure 7

(captions not provided) 


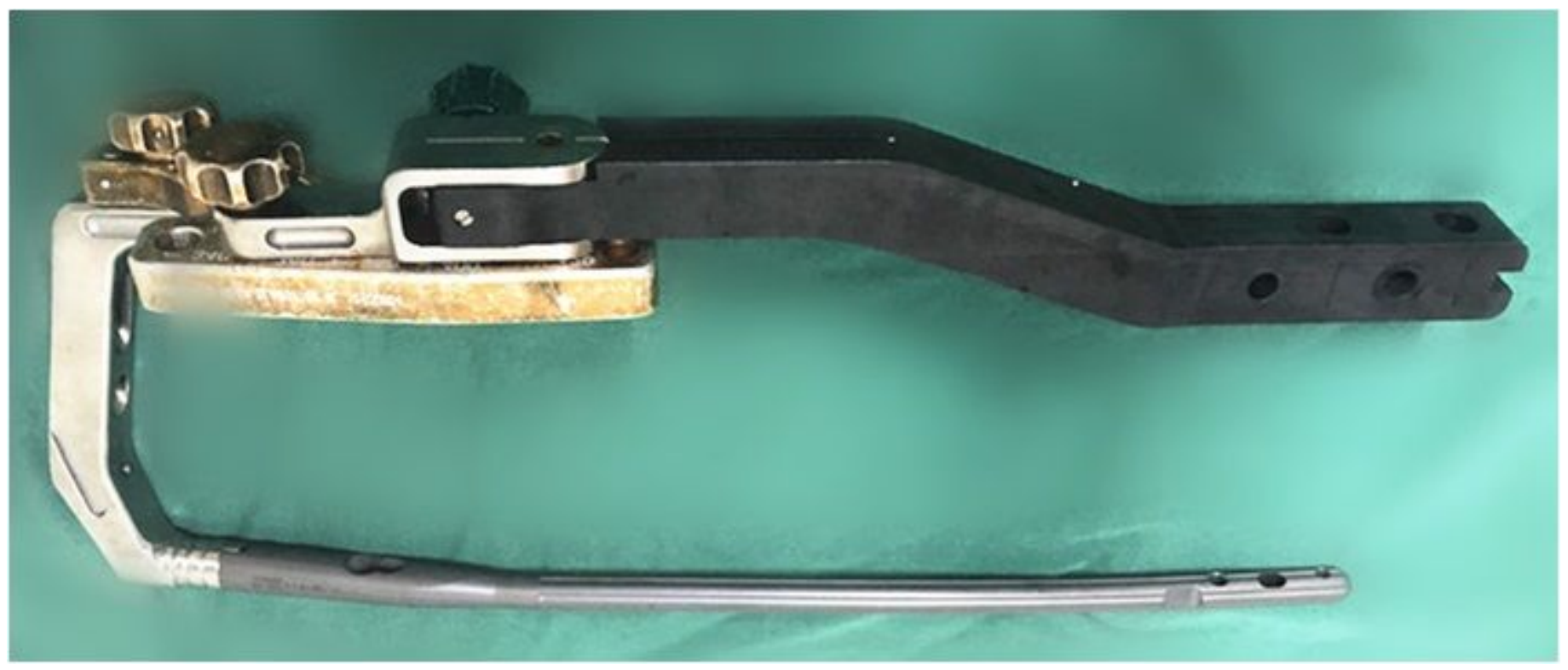

Figure 8

(captions not provided)

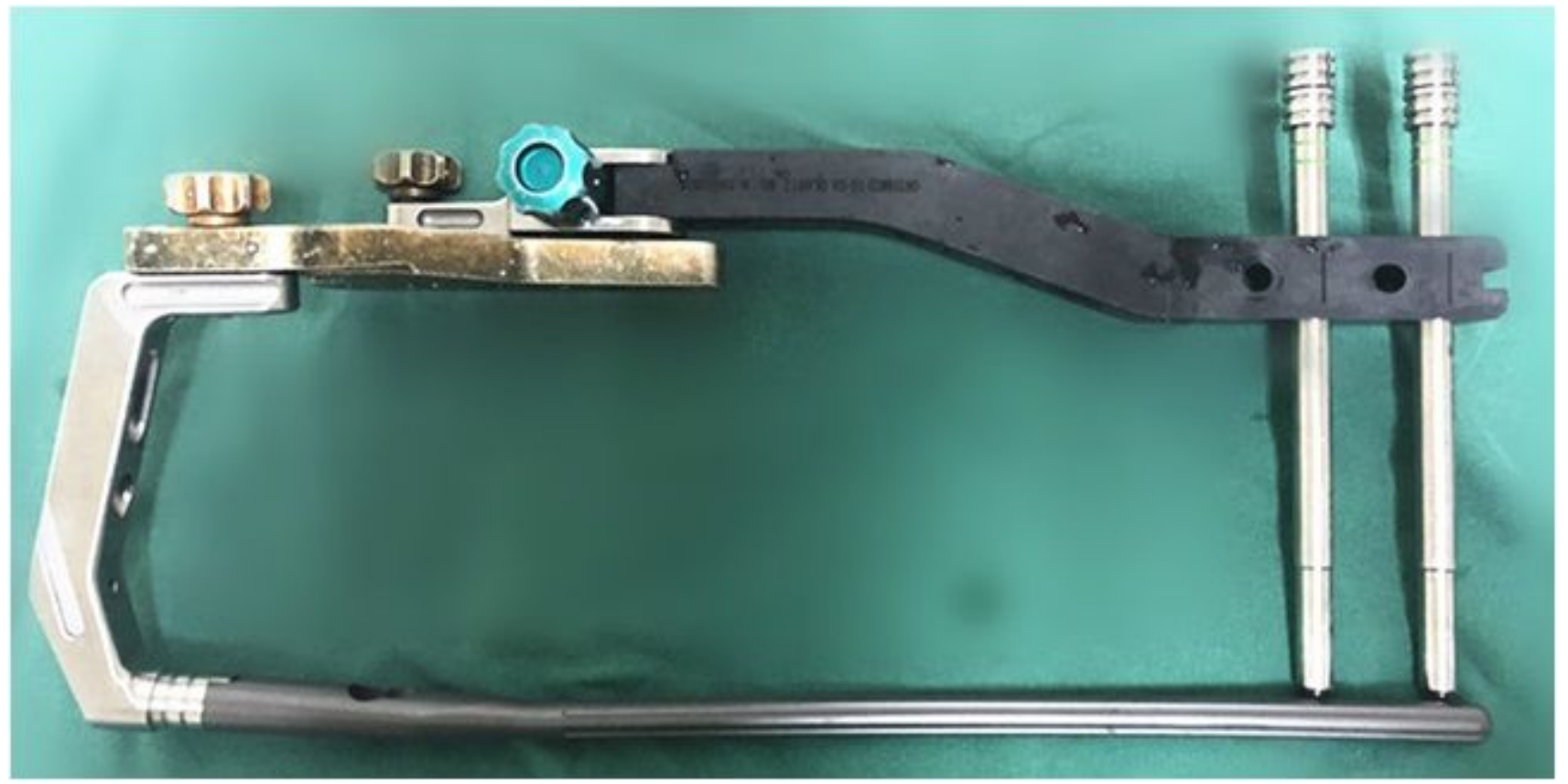

Figure 9

(captions not provided) 


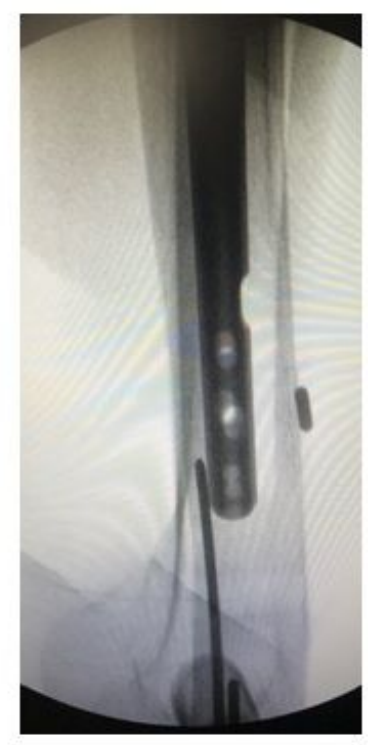

Figure 10

(captions not provided) 


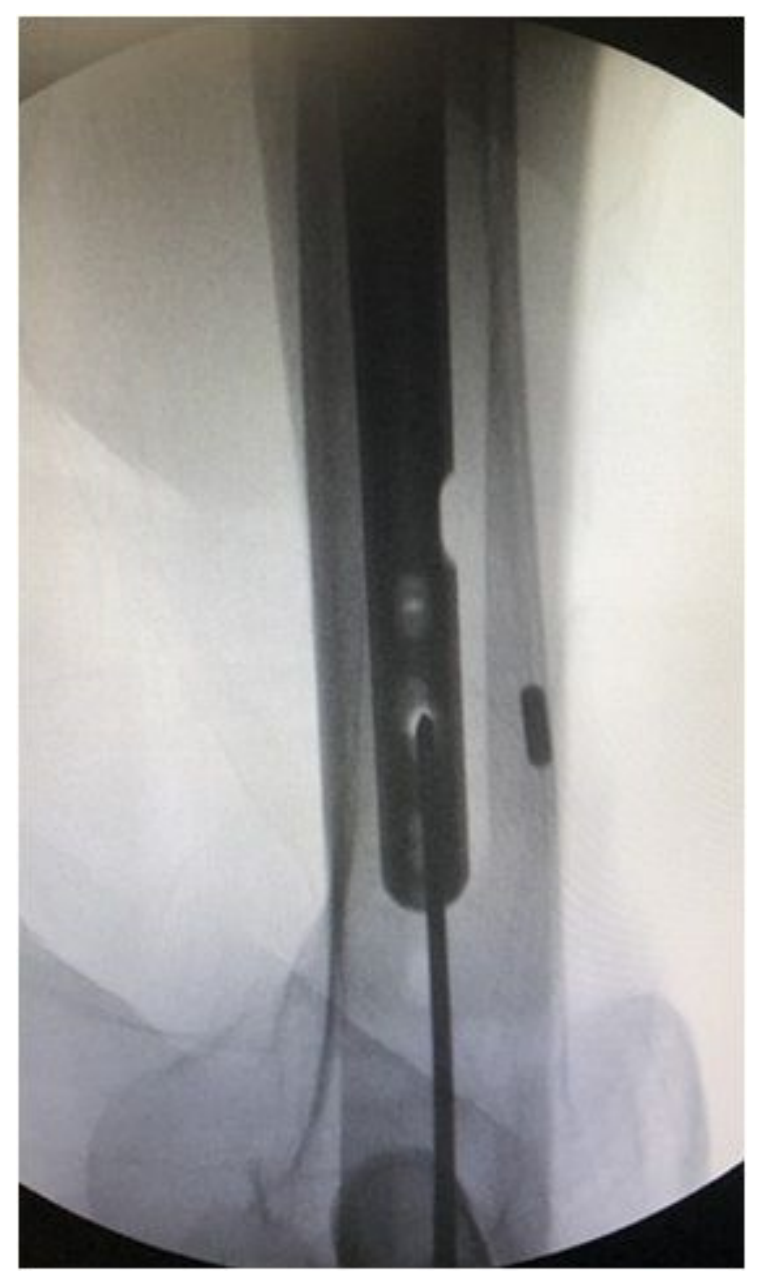

Figure 11

(captions not provided) 

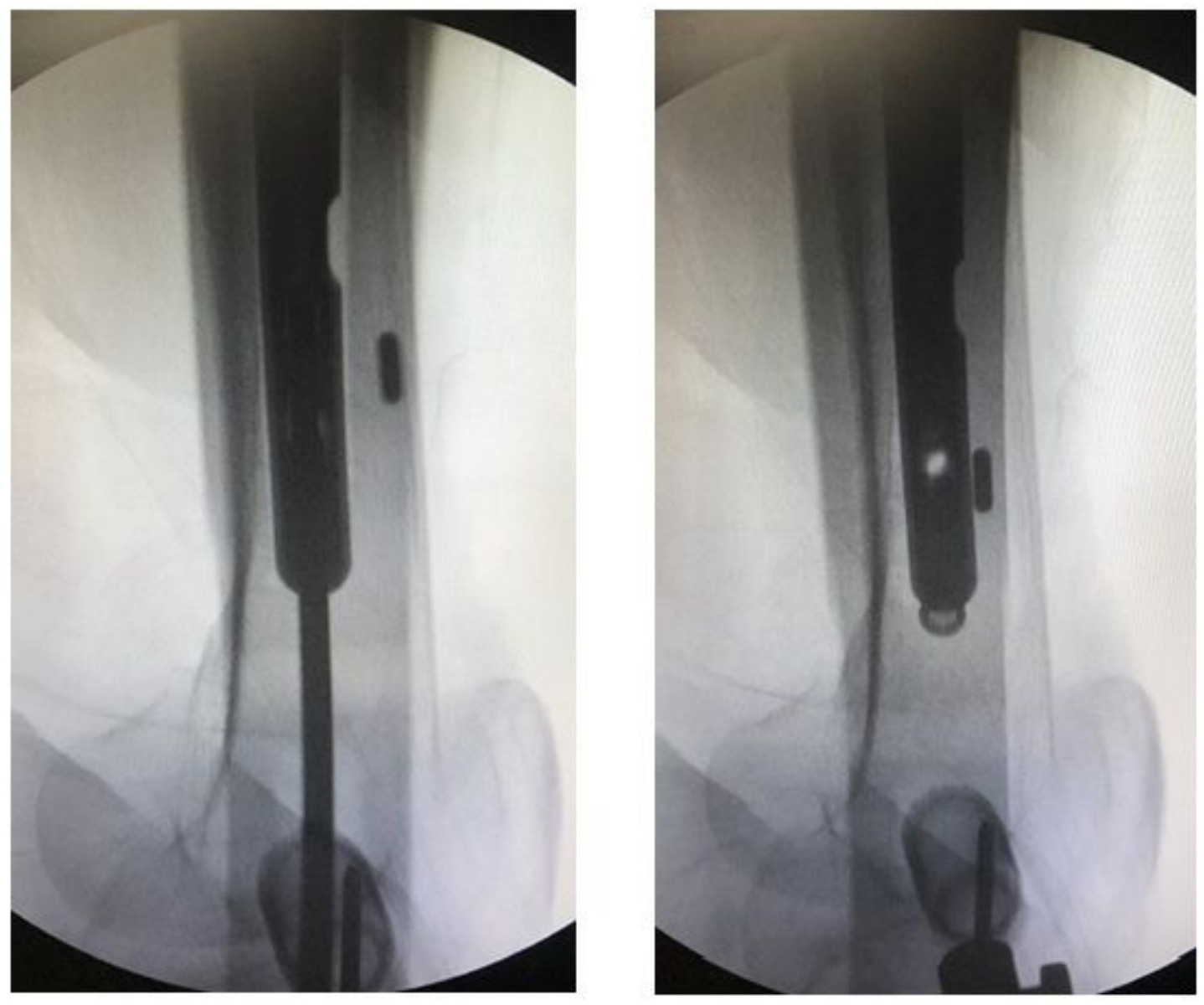

Figure 12

(captions not provided) 

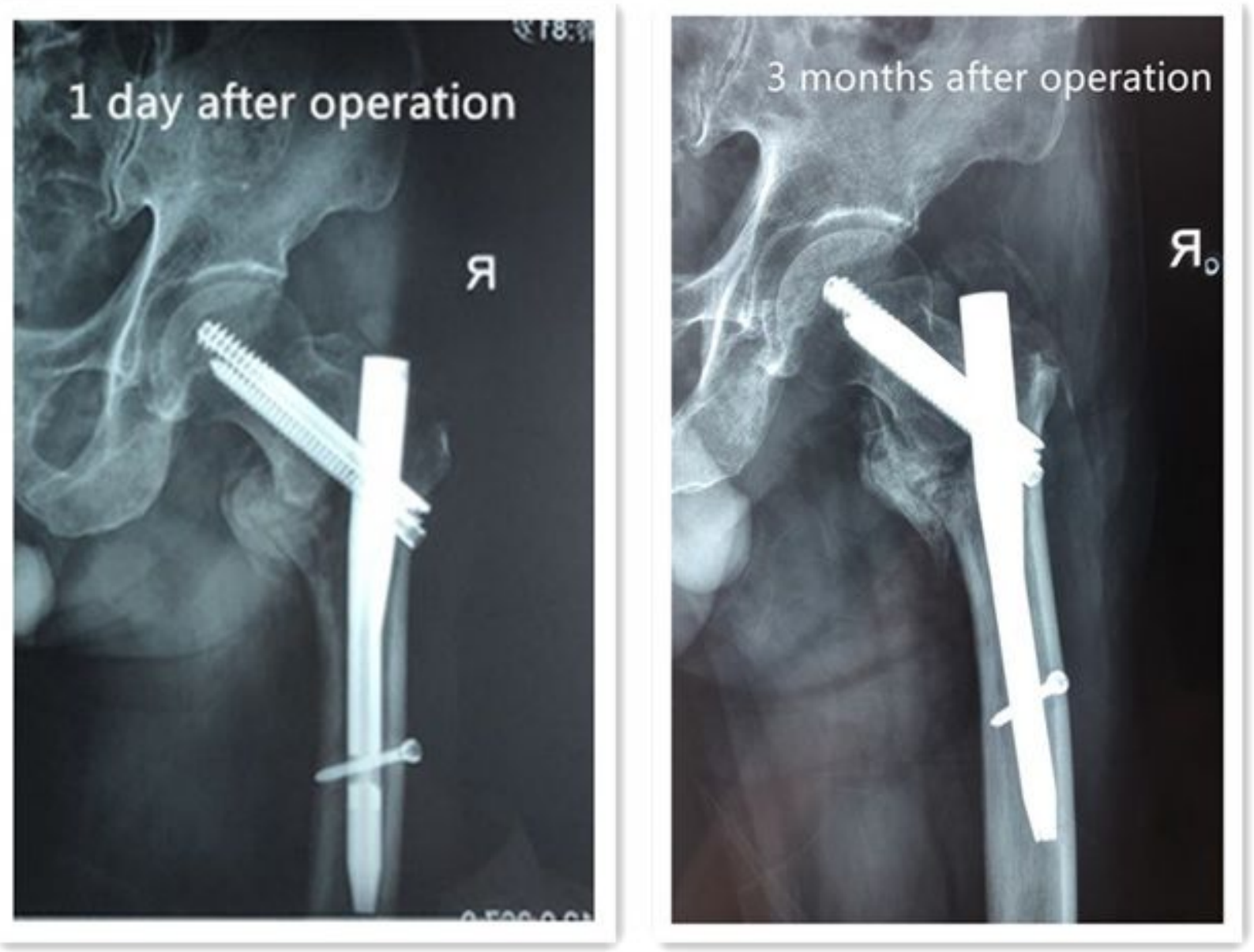

Figure 13

(captions not provided)

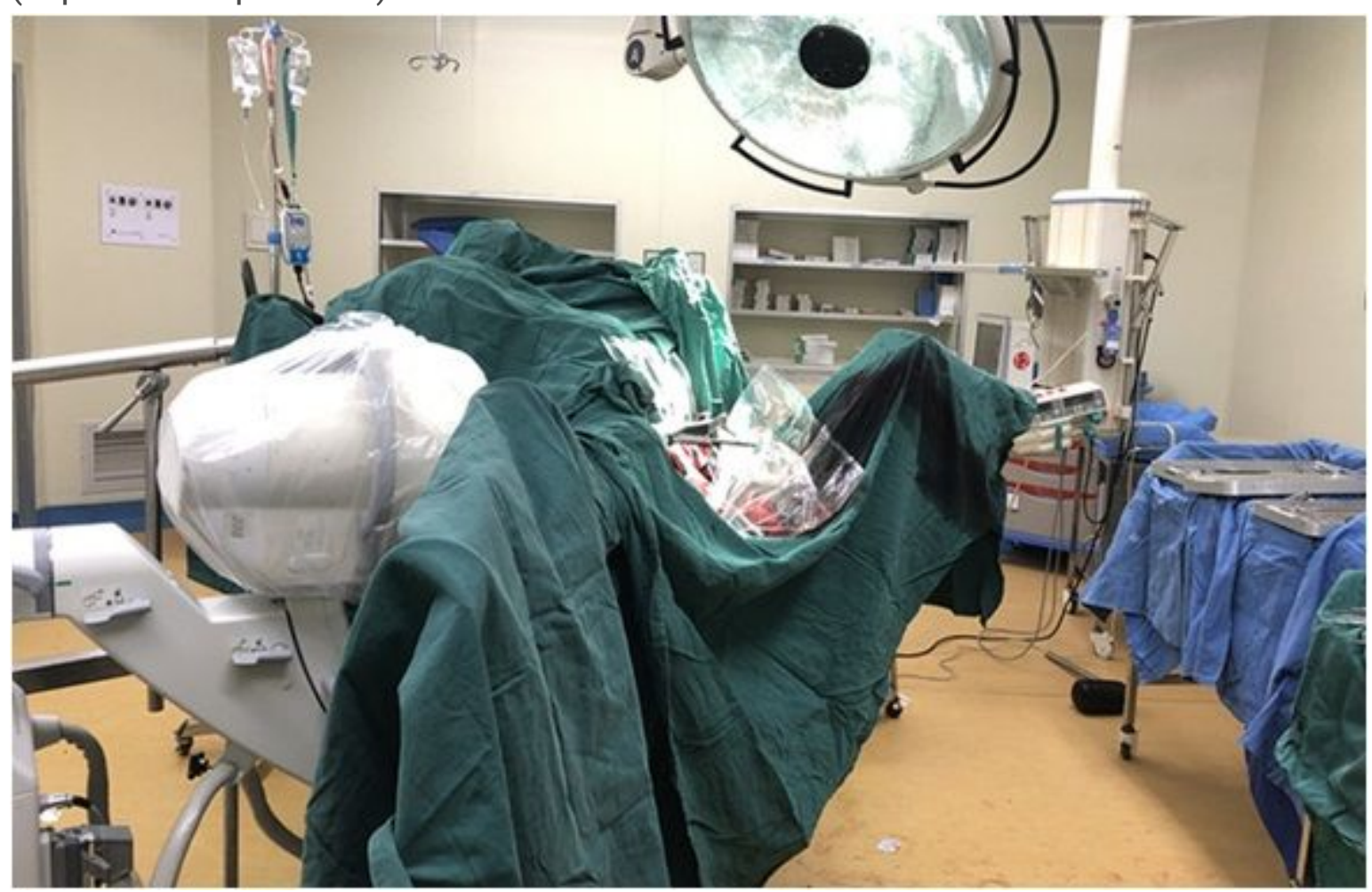


Figure 14

(captions not provided)
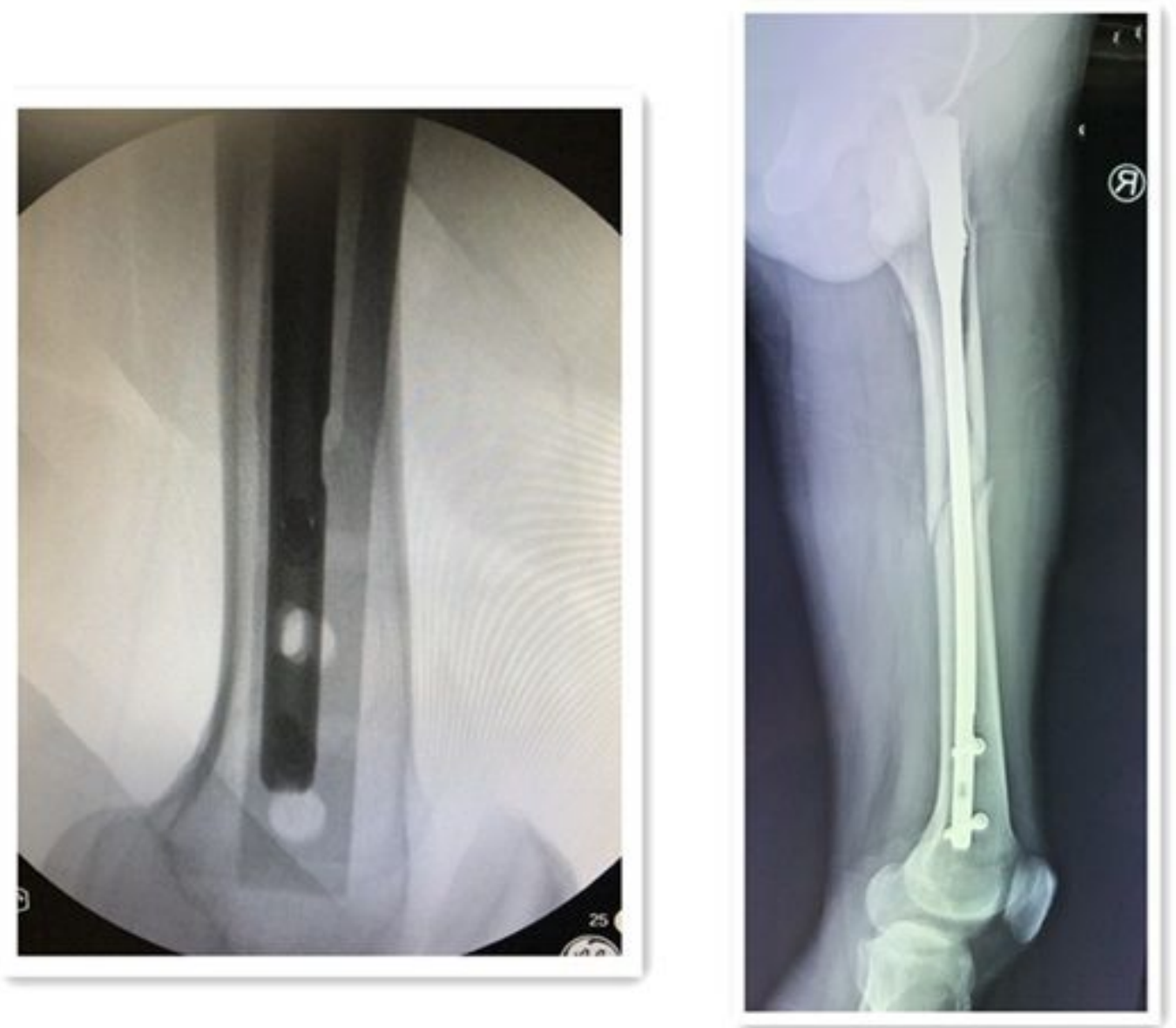

Figure 15

(captions not provided) 

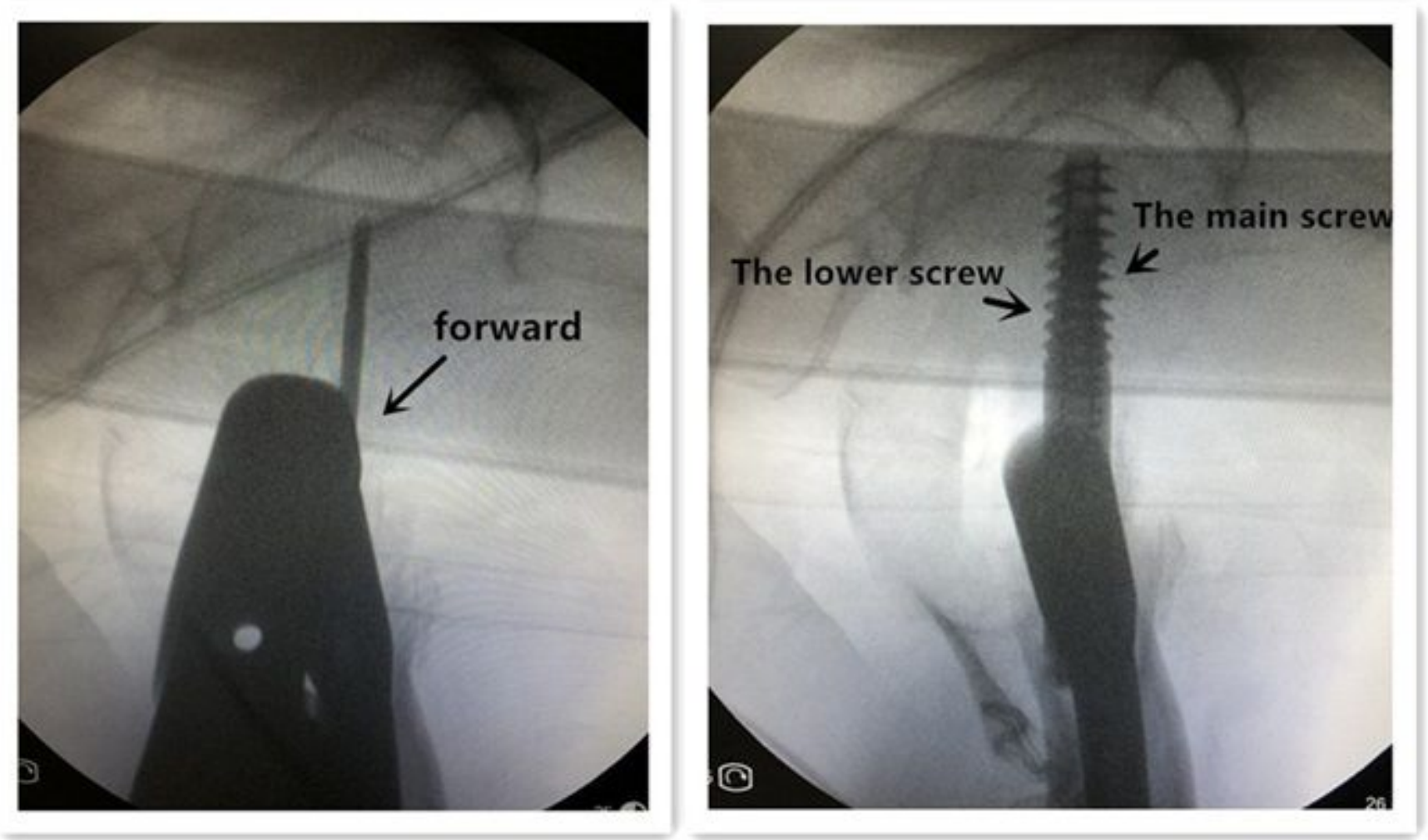

Figure 16

(captions not provided) 